\title{
Experiências de Uso de Mídias Interativas como Suporte para Autoria e Construção Colaborativa do Conhecimento
}

\author{
Jorge Ferreira Franco (1)1,2 \\ Nilton Ferreira Franco (2) 3 \\ Sandra Regina Rodrigues da Cruz (3)2 \\ Roseli de Deus Lopes (4)1 \\ 1-Laboratório de Sistemas Integráveis (LSI -USP) \\ Av. Prof. Luciano Gualberto, travessa 3, no 158 - São Paulo - CEP: 05508-970 \\ 2-PMSP-SME - Av. Interativa $n^{\circ} 100$ - São Paulo - SP - CEP: 02820-020 \\ 3-Universidade Presbiteriana Mackenzie - Pós Graduação em Arte e História da Cultura \\ Rua Piauí, 143 - São Paulo - CEP: 01241-001 \\ \{jfranco,roseli@lsi.usp.br\},niltonfranco.net@ig.com.br, masalelu@ig.com.br
}

\section{Resumo}

Neste artigo, experiências educacionais colaborativas apoiadas por computador, com suporte da combinação de teorias de aprendizagem, tecnologias padrão da Internet, sistemas de visualização de informação, tanto quanto de instrumentos e arquivos multimídia de baixo custo na construção de conhecimento dos indivíduos, e na produção de conteúdo digital e curricular são apresentadas. $\mathrm{O}$ desenvolvimento das experiências educacionais demonstra que através do uso da sinergia entre educação e tecnologia na aprendizagem formal e informal dos indivíduos, é possível contribuir efetivamente para desenvolver os processos de inclusão digital e ampliar o acesso à arte e a cultura de modo flexível, dinâmico, expansível e sustentável.

Palavras chave: educação, tecnologias padrão da internet, visualização de informação, construção de conhecimento

\begin{abstract}
In this article, collaborative computer supported learning experiences with support of the combination among learning theories, the Internet standard technologies, low cost information visualization systems as well as multimedia tools and files on individuals'

nowledge construction and digital and curriculum content production are presented. The development of the learning experiences shows that through the use of the synergy between education and technology on individuals' formal and informal learning, it is possible to contribute effectively to develop the processes of digital inclusion and improving individuals' access to arts and culture within a flexible, dynamic, expansible and sustainable mood.
\end{abstract}

Key words: education, internet standard technologies, information visualization, knowledge construction

\section{Introdução, Problematização e Fundamentos do Trabalho}

A constante queda nos custos de hardware e maior acesso à Internet e suas tecnologias têm ampliado a possibilidade de uso dos sistemas de visualização de informação e multimídia com maior intensidade e efetividade nos processos de ensinoaprendizagem, tanto do ponto de vista qualitativo quanto do quantitativo. Essa ampliação do acesso aos meios digitais embasa este trabalho desenvolvido de modo colaborativo e cooperativo com suporte de instrumentos e arquivos multimídia, tanto quanto de espaços físicos e virtuais diversos. Também, engloba aspectos da interação entre percepção e 
cognição através de recursos computacionais diversos (Minsky, 2005), e mediação, com origem, por exemplo, na reunião de artistas e pesquisadores desenvolvendo trabalhos que aproximam espaços materiais "reais" e eletrônicos "virtuais" (Anders, 1998 p.171, 177).

Essa mediação e interações de ambientes reais e virtuais acrescentam ganhos ao trabalho pedagógico para estimular a percepção e cognição dos indivíduos, e incrementar as idéias de trabalho colaborativo, servindo como suporte para a construção de projetos inter e transdisciplinares que, por exemplo, tenham como base de desenvolvimento tecnologias padrão da Internet em combinação com fundamentos de produção artística e cultural (Laybourne e Goldberg, 1998 p.258, 259; Laybourne e Bell, 1998 p. 265-276; Laybourne e White, 1998, 264 p.; Perlin. 2007). Exemplos de projetos são o Improv in (Goldberg, 2003) e NICE (Roussos et al, 1999). Tal combinação de técnica, arte e cultura, e exemplos de projetos sugerem usá-los como referência no desenvolvimento das habilidades de aprender a aprender, ler, escrever, pesquisar, programar, comunicar e de pensar dos indivíduos de modo mais reflexivo e crítico, com maior autonomia em diversos tempos e espaços (Franco et al, 2006d).

Além de estimular aprendizagem individual continuada mais autônoma, o conhecimento transdisciplinar adquirido tem influenciado para fomentar e transformar pesquisa e desenvolvimento de currículo em processos mais interativos, inclusivos, cooperativos, colaborativos, e "contextualizados" conforme (Morim, 2002b, p. 29) citado por (Augusto e Caldeira, 2007). A participação dos indivíduos nestes processos demonstra que os instrumentos e formas de aprendizagem propostos são propícios para envolver a comunidade escolar e do entorno de modo dinâmico, flexível, expansível e sustentável (Franco et al, 2006a; Franco et al, 2006b), tanto quanto para validar a construção, uso e reuso de uma arquitetura pedagógica e técnica, flexível e aberta descrita em (Franco ET al, 2006a), que embasa a construção de conhecimento colaborativo dos indivíduos na combinação entre as ciências, educação, tecnologia, arte e cultura (Franco et al, 2006c).

O uso de uma arquitetura pedagógica e técnica, flexível e aberta, e o embasamento transdisciplinar na construção individual e colaborativa de conhecimento têm contribuído para que se aprenda a tratar problemas como os da educação em valores, de desenvolver formas dialógicas e democráticas de resolução de conflitos cotidianos e problemas sociais através da busca de articulação entre os conhecimentos populares e científicos dando um novo sentido à escola. Assim, tais ações e reflexões têm servido como base para conectar a escola com a vida das pessoas e desenvolver uma formação ética para a cidadania através da educação (Araújo, 2003, p. 58-59).

Portanto, as experiências deste trabalho podem contribuir para ampliar as possibilidades de reflexão sobre o uso da convergência entre teorias de aprendizagem, sistemas eletrônicos e mídias interativas como suporte para a construção do conhecimento dos indivíduos e diminuição dos problemas de alto índice de analfabetismo tradicional e digital, e conseqüente dificuldade de acesso à arte e à cultura e sua compreensão (INAF, 2005). E mais, o uso dessa convergência tem contribuído como um modo de minimizar o problema da necessidade permanente de formação qualitativa dos cidadãos em geral, e de modo mais específico de professores para uso efetivo das tecnologias da informação e da comunicação - TIC no desenvolvimento curricular (Franco, 2001; Barreto, 2003, 109-118), para além do nível rudimentar descrito na pesquisa (INAF, 2005).

Experiências com a formação de educadores, como por exemplo, a do Núcleo de Informática Aplicada à Educação - NIED da Unicamp, tanto quanto as experiências educacionais empíricas e interativas que têm sido vivenciadas nos ambientes educacionais objetos de pesquisa e de aplicação descritos posteriormente neste artigo embasam que a formação dos indivíduos para viverem na sociedade da informação precisa ser realizada para além da passagem de informações sobre o uso pedagógico da informática. Pois segundo Valente (2003, p. 3-4), no que se refere à formação de professores, a formação 
deve oferecer condições para que os educadores construam conhecimento sobre técnicas computacionais e entendam porque e como integrar o computador em sua pratica pedagógica. Com formação adequada, o conhecimento pedagógico dos educadores aliado ao seu desenvolvimento técnico tende a propiciar, por exemplo, o engajamento de estudantes no aprender a investigar e manipular diretamente tecnologias padrão da Internet para produção de conteúdo digital de modo integrado ao desenvolvimento curricular no contexto escolar e fora dele. Estas ações tendem a servir de base para a construção de um processo de educação continuada, mesmo em locais onde os indivíduos vivam em situação sócio-econômica desfavorável, mas tenham acesso aos computadores e às tecnologias de baixo custo fora do ambiente escola (Franco e Lopes, 2005; Franco et al, 2006d).

As experiências demonstram que as aplicações de tecnologias padrão da Internet na educação podem dar suporte ao uso de linguagem referente à cultura visual, na interpretação crítica, e construção de conteúdo digital. O domínio mútuo da linguagem visual e dos meios de produção de conteúdo tende a ampliar as possibilidades de compreensão sobre seu uso, tanto quanto de comunicação entre educadores e estudantes. Assim, é necessário vincular linguagem e meio de produção "à reflexão crítica sobre as diferentes tradições históricas, filosóficas e culturais que servem de guia para construir "representações" mediadoras de significados em diversos tempos e espaços dessa construção cultural denominada história” (Hernández, 2000, 50-51).

Recente trabalho que une a combinação entre arte, tecnologia, história, cultura na construção de uma representação de parte do patrimônio histórico cultural da cidade de São Paulo é o projeto Modelagem descrito e ilustrado em (DPH, 2007; LSI, 2007; Cabral et al, 2007). Outro trabalho que embasa a construção deste artigo é a experiência colaborativa dos autores 1 e 2, que através de recursos de visualização de informação e computação gráfica de baixo custo projetaram um ambiente virtual - AV com suporte do conceito de objetos educacionais e técnicas de realidade virtual - RV não imersiva em (Kiner, 2007), com o uso de tecnologias padrão da Internet a partir de uma investigação sobre a Aldeia de Carapicuíba, que é um dos patrimônios culturais brasileiros, e a obra artística e cultural de Alfredo Volpi figura-1 (Franco et al, 2005).
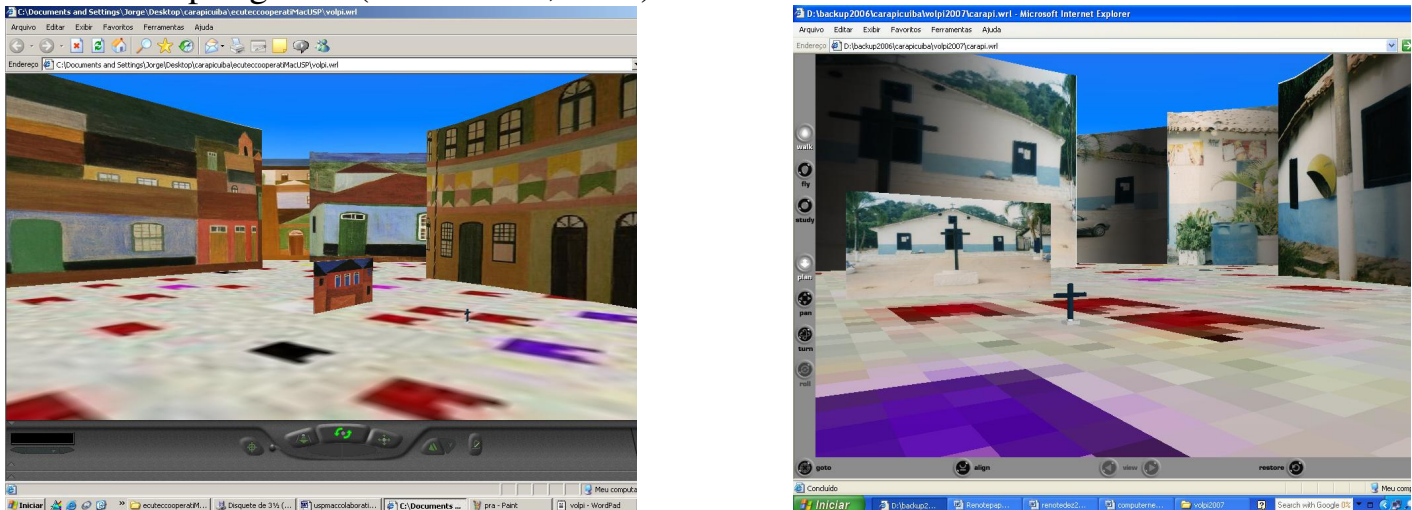

Figura-1 - Ambiente virtual desenvolvido com a linguagem VRML e texturas provenientes da obra de Alfredo Volpi e de fotos tiradas durante pesquisa de campo.

Essas experiências, reflexões, aplicações e resultados apresentados com o uso da tecnologia em escola pública, segundo Leu (2000) citado por (Taberosky, 2004, p. 160), contrapõem as investigações que, em geral, avaliam o impacto do uso das TIC fora do contexto escolar, já que, graças aos computadores pessoais, a tecnologia eletrônica está mais presente em certos lares de classe média do que nas escolas. No que se refere ao problema da necessidade de formação docente qualitativa para uso efetivo das TIC, essas experiências encontram respaldo investigativo e reflexivo no trabalho de (Barreto, 2003, 109-118), com "relação à formação docente, tendo em vista a relação TIC-EAD neste 
tempo e espaço" e a aplicação com qualidade do conhecimento adquirido no cotidiano escolar.

Por outro lado, Segundo Leher (1998) citado por (Barreto 2003, p. 112), a formação de professores que está no elenco das "condicionalidades" para a concessão de empréstimos aos paises do Terceiro Mundo tem sido "deslocada e ressignificada" como certificação, alvo, por exemplo, de programa específico da Secretaria de Educação a Distância (SEED-MEC): o Proformação, destinado aos professores no local de serviço. Os programas de educação a distância têm oferecido formação especifica e pedagógica aligeirada com visão de que as TIC possam dar conta, de modo econômico e eficaz do desenvolvimento de certas habilidades especificas dos indivíduos (Barreto 2003, p. 112). Pesquisa recente indica que mesmo em países do Primeiro Mundo onde a cultura de ler, escrever e pesquisar com autonomia, é quantitativa e qualitativamente superior a do Terceiro Mundo, há dificuldades na estruturação dos cursos de educação a distância, tais como a perda da qualidade das interações sociais que ocorrerem na sala de aula; sobrecarga no trabalho docente; baixo nível de satisfação e interesse; problemas técnicos e de logística; e a necessidade dos indivíduos de perseverar em sua trajetória de aprendizagem. A pesquisa indica como resolução para os problemas alternar a formação entre momentos presenciais e on-line com qualidade (Hirschheim, 2005, p. 97 -101).

Entretanto, conforme tem sido vivenciado na construção deste trabalho no cotidiano de um laboratório de informática de uma escola pública de ensino fundamental, desde 2002, em (Franco e Lopes, 2004), propiciar o domínio técnico das TIC pelos docentes e discentes, e seu uso no desenvolvimento curricular e nas atividades pessoais e profissionais fora do ambiente escola, é que pode respaldar e certificar a excelência da experiência de ensino-aprendizagem com suporte das TIC em longo prazo. Para tanto, é necessário que as políticas educacionais de formação da comunidade escolar sejam abrangentes e permanentes, favoreçam interações presenciais e virtuais diversificadas, e tenham um planejamento de longo prazo. Um exemplo é plano Response To Intervention (RTI, 2006), que visa criar ambiente educacional de excelência para todas as crianças através de ações colaborativas envolvendo diversos seguimentos da sociedade. Assim, é fundamental para elevar a qualidade do sistema de ensino, diminuir a "divisão digital" através do desenvolvimento humano e apropriação das TIC com efetividade (Afonso e Soares, 2005), e formar indivíduos/professores capazes de se apropriarem das TIC, como usuários/produtores conforme recomenda Castells (2005, p. 69), ao invés de apenas serem consumidores de mercadorias postas cada vez mais baratas, acessíveis e de manuseio mais simples em (Barreto, 2003, p. 117).

Ao longo do tempo, as experiências de ensino-aprendizagem com uso das TIC têm ajudado no processo de mudança de atitude de educadores e estudantes no sentido de engajarem-se em novas formas de aprendizagem e perseverarem para que a mudança de paradigma de ações educacionais tenha êxito. Pois, parafraseado Tajra (2001 p. 127), entre o momento da percepção da necessidade de mudar e ter resultados com as mudanças adotadas, existe um "espaço/intervalo" em que os indivíduos processam as mudanças de paradigmas. Embora, em geral, o processo de aprendizagem seja doloroso, o tempo e a experiência possibilitam aos indivíduos sentir maior segurança, e assim alcançar patamar mais elevado de desenvolvimento pessoal e profissional, com forte suporte de conhecimento desenvolvido e compartilhado em (Senge, 1997, p.123-145), e do amplo conceito que é o de capital intelectual, que em resumo, “(...) abrange os conhecimentos acumulados por uma organização, relativos a pessoas, metodologias, patentes, projetos $e$ relacionamentos (...)” em (Santiago e Santiago, 2007, 38 p.).

O foco deste trabalho está no uso de linguagens padrão da Internet como ferramentas de autoria, contribuindo para a ampliação das habilidades cognitivas gerais e específicas dos educadores/educandos, estimulando a criatividade e o protagonismo juvenil; 
desenvolvendo a iniciativa para a resolução de problemas das diversas ciências com suporte de sistemas de visualização de informação e computação gráfica (Cunnigham, 2007, p. 331378; Brutzman, 2007, p. 1-36); promovendo o domínio das inovações tecnológicas como uma fonte de inspiração e conscientização dos indivíduos sobre a importância de ler e escrever adequadamente, e de responsabilidade com a formação do capital humano e social (Dolabela, 2003) para compreender, usufruir e influir com consciência nos processos de produção de conteúdo tradicional e digital. Assim, propiciar condições para o desenvolvimento dos talentos e qualificação das pessoas para interagirem com os novos desafios e constantes mudanças do mundo contemporâneo, de modo mais autônomo e sustentável, dentro de um processo de propiciar desenvolvimento socioeconômico, democratização de acesso, apropriação e uso das TIC (Vieira, 2003, p. 131-149; Hippel, 2007, Tapscot e Williams 2007; Balboni, 2006).

$\mathrm{O}$ artigo segue com apresentação de algumas experiências de ensino aprendizagem com aplicação de mídias interativas como suporte para a autoria e construção de conhecimento através de casos de uso, avaliação e conclusão.

\section{Casos de Uso e Estratégias de compartilhamento de Saberes Fundamentos Pedagógicos e Técnicos}

O desenvolvimento das experiências está respaldado pelo suporte de teorias e metodologias relativas ao desenvolvimento cognitivo dos indivíduos em combinação com aspectos técnicos relativos ao mundo digital formando uma arquitetura pedagógica e técnica flexível e aberta descrita em (Franco et al, 2006a). Em resumo a arquitetura envolve o construcionismo de Papert e o construtivismo de Piaget; as idéias de Paulo Freire (2004) sobre ensino-aprendizagem dialógico, contribuindo para uma relação mais horizontal entre educadores e educandos com base na sinergia entre o respeito pela liberdade de expressão, humildade e amor em combinação com o rigor metodológico; a pedagogia de projetos e a importância da experiência, que estão relativas às idéias de John Dewey; o conceito da zona de desenvolvimento proximal - ZPD de Vygotsky; a pedagogia empreendedora em (Dolabela, 2003, p. 91-93); a convergência dos fundamentos de psicologia e aprendizado experimental com as técnicas do modelo espiral de construção de software em (Bohem, 1998; ICEL, 2007); a teoria de Gardner sobre as múltiplas inteligências; e o conceito de design emergente, que segundo (Cavallo, 2000) dá apoio teórico às ações que englobam tecnologia e administração na condução de transformações educacionais. O pesquisador, também, reflete sobre o quão importante é manter-se o equilíbrio entre esses dois produtos da era digital para que haja sucesso nos projetos. Ele visualiza, com base empírica (Cavallo, 2000), que a convergência tecnologia e administração em sinergia com os conceitos educacionais dos pensadores da era pré-digital dão suporte adequado para realizar o que os pensadores, como Dewey e Freire, sabiam o que, e como fazer, mas não tinham meios para fazê-lo.

Com relação ao trabalho técnico, o referencial para desenvolvimento das experiências vem de padrões e conceitos como os dos objetos de aprendizagem, IMS e SCORM em (Wikipedia, 2007; Dutra e Tarouco, 2006; Ferreira, 2004); tecnologias padrão da Internet, ex: navegadores Internet Explorer ${ }^{\mathrm{TM}}$, Cosmo Player ${ }^{\mathrm{TM}}$ e Cortona ${ }^{\mathrm{TM}}$, a linguagem Virtual Reality Modeling Language - VRML que descreve mundos virtuais em 3D (Ames et al, 1997), tutoriais e referências na Internet (UFRGS, 2007, Cortona, 2007; VRML sourcebook on-line, 2007; Barros, 2004); técnicas de realidade virtual e sua aplicação, entre outras, no desenvolvimento educacional (Pinho, 1996).

\section{Caso de Uso 1}

A aplicabilidade dos conceitos pedagógicos e técnicos abordados até este trecho do artigo no cotidiano dos indivíduos tem se mostrado viável. É nesta perspectiva que o exemplo a seguir é apresentado.

\section{Contexto}


Aluno do $8^{\text {a }}$ ano do ensino fundamental II, da EMEF Ernani Silva Bruno, M1 acompanha o desenvolvimento dos trabalhos com tecnologias padrão da Internet realizados pelos autores 1 e 3, no laboratório de informática da referida escola, desde de 2003, quando seu irmão, o aluno M2 e um grupo de alunos começou a participar das primeiras experiências com tais tecnologias (Franco e Lopes, 2004). Em 2005, M1 solicitou ao autor1 que explicasse como formatar um ambiente em 3D utilizando as mesmas técnicas que seu irmão usava para produzir conteúdo digital. Naquele tempo M1 não tinha computador em casa e embora tenha participado de duas a três oficinas de VRML no laboratório de informática, não foi o suficiente para ganhar autonomia para produzir conteúdo, mesmo tendo se mostrado satisfeito com o aprendizado. Em 2006, M1 participou de mais quatro oficinas no laboratório de informática após ter acesso a um computador em sua casa. M1 solicitou que fosse cedido a ele material técnico via CD para configurar um navegador que interpretasse a linguagem VRML, pois não tinha acesso a Internet naquele período. Porém após ter configurado a máquina e treinado com a linguagem durante algum tempo usando tutoriais, seu computador quebrou.

\section{Experiência de Uso da VRML no Desenvolvimento Curricular}

Em 2007, M1 visita ao laboratório para pesquisar sobre Charles Chaplin e diz ter que desenvolver uma apresentação para a professora de língua portuguesa. É orientado a produzir um AV para visualização de informação e comunicação de sua investigação sobre Chaplin. Entretanto, M1 relata os problemas ocorridos com seu computador, conforme descrito no contexto inicial. Então, solicita ajuda para relembrar os procedimentos de como salvar um arquivo e ativar a percepção espacial necessária para realizar as transformações virtuais dos objetos criados e /ou reutilizados. Realiza-se então uma oficina interativa que dura por volta de 40 minutos. Durante a interação, pesquisa-se uma textura na Internet, lê-se código VRML e modela-se um pequeno protótipo de museu virtual que inspira M1 a refletir e retomar seu aprendizado com autonomia, e apresentar um modelo de sua autoria após cinco dias figura-2.
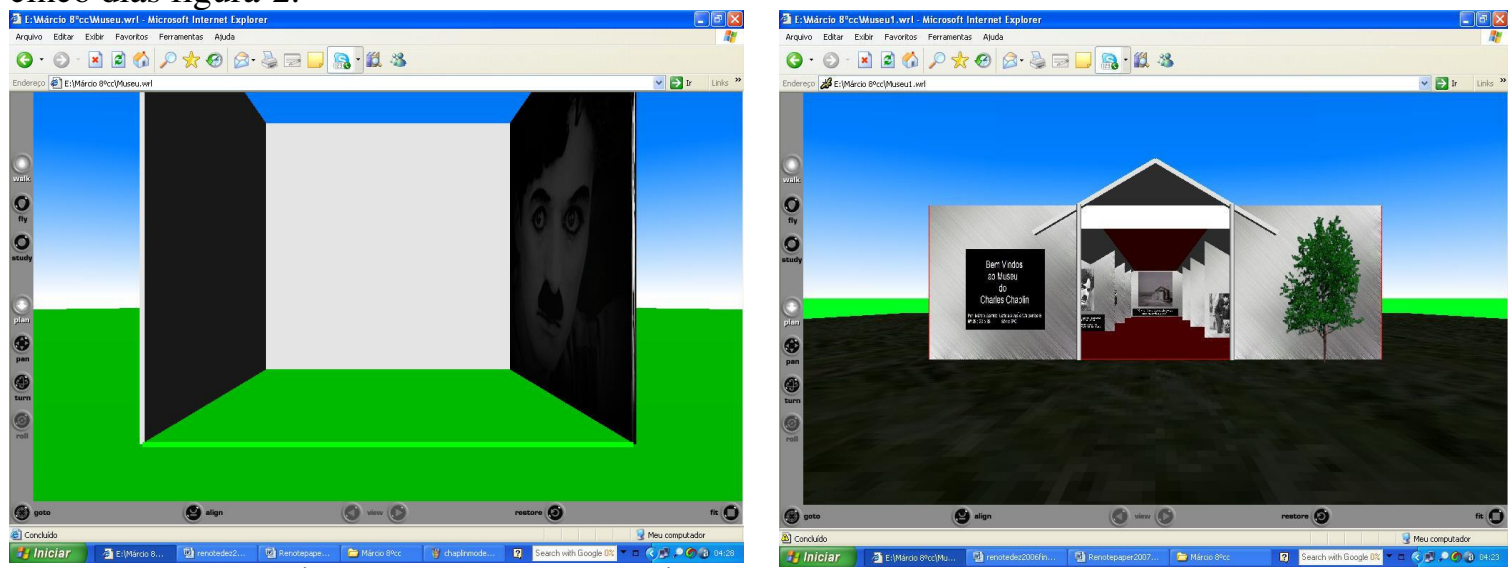

Figura-2 - À esquerda modelo inicial. À direita modelo interativo 3D criado por M1, com uso

da

característica da linguagem VRML chamada 'move texture'.

M1 explica o desenvolvimento do ambiente à direita em forma de corredor, como representativo de geometria apropriada para resolução de um problema com recurso de computação gráfica conforme (Cunnigham, 2007, p. 332). Pois, através de sua percepção constata que é mais fácil apresentar a pesquisa em termos de linha de tempo com paredes virtuais dos dois lados do corredor, do mesmo modo como são mostradas as paredes da entrada do museu, a do lado esquerdo com uma textura e a outra do lado direito com a árvore mais à frente. Sendo informado da possibilidade de colocar filme como textura, após pesquisa na Internet (YouTube ${ }^{\mathrm{TM}}, 2007$ ), M1 selecionou trechos referentes ao filme Tempos Modernos, da obra de Charles Chaplin, com o objetivo de incrementar o trabalho. Por 
questões técnicas, não foi possível baixar os trechos selecionados. Entretanto, através de conversa informal, entre os autores 1 e 2 sobre o AV desenvolvido por M1, o autor2 colaborou emprestando o filme que foi editado pelo autor1, de acordo com as pesquisas de M1, utilizando uma placa de captura de baixo custo, PixelView ${ }^{\mathrm{TM}}$. Assim, as características de trabalho colaborativo e cooperativo foram sendo implantadas ao projeto de M1.

A estrutura do AV está sendo reusada para dar suporte multimídia à pesquisa do autor2, relativa à arte e transformações sociais.

Além de apresentar o trabalho com inovação e qualidade para a professora e colegas de sala, M1 mostrou seu projeto ao pesquisador Marcelo Zuffo do Laboratório de Sistemas Integráveis - LSI, USP, por ocasião de sua visita a escola em 22/06/2007. M1 relatou para o pesquisador o processo de criação do $\mathrm{AV}$, praticando, também, suas habilidades de comunicação conforme discutido em (Roussos et al, 1999). Certamente que por ser a primeira vez que se encontraram, a aproximação e a interação deles foram facilitadas devido ao conhecimento comum e domínio mútuo de linguagem visual e dos meios de produção de conteúdo digital no que diz respeito à familiaridade com as técnicas de RV.

Caso de Uso 2 - Compartilhando Conhecimento e outra Comunidade Escolar

A partir de abril de 2007, em suas aluas de matemática, o autor 3 apresenta aos seus estudantes do ensino médio de uma outra comunidade escolar da rede estadual de ensino, na cidade de Caieiras, alguns modelos 3D em VRML construídos por alunos do $7^{\circ}$ ano, da escola Ernani, em 2005, durante o desenvolvimento curricular e oficinas de aprimoramento de conhecimento técnico envolvendo a sinergia entre ciência, arte, cultura e tecnologia (Franco et al, 2006d). Com a autonomia desenvolvida ao longo das experiências, o autor3 decidiu oferecer, usar e compartilhar seus conhecimentos de VRML como forma de motivar os alunos a se aplicarem mais nas aulas e aprenderem conceitos matemáticos, até então bastante abstratos, de modo mais concreto e proativo.

Embora no laboratório da escola tenha 8 computadores funcionando para 40 alunos, a professora se diz satisfeita em ver o engajamento dos estudantes em tentar aprender a linguagem para desenvolver novas criações para além das que foram apresentadas como exemplo, conforme pode-se constatar no relato do recorte mostrado na figura-3.
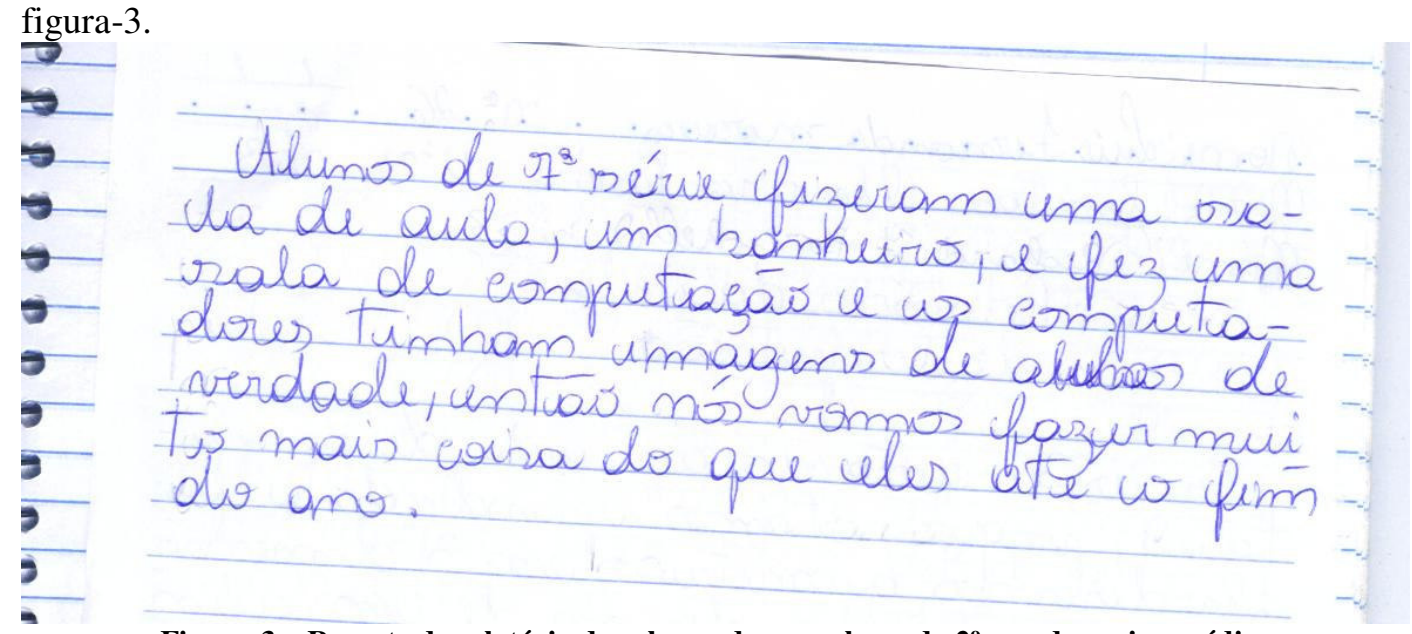

Figura-3 - Recorte de relatório dos alunos de uma classe do $2^{\circ}$ ano do ensino médio

Essa experiência contribui para comprovar os argumentos sobre aprender a usar na educação linguagens e técnicas de visualização de informação e comunicação que são familiares aos alunos e que possam contribuir para motivar os educandos a aprender na escola e para além dela. Certamente que para atingir este objetivo propiciar aos educadores(as) formação pedagógica e técnica qualitativa e diversificada permanentemente, 
é condição fundamental em tempos de transformações constantes como as atuais, conforme argumentado na seção de introdução, problematização e fundamentos do trabalho.

\section{Avaliação}

Considera-se que a convergência entre as teorias e estratégias educacionais, os aportes tecnológicos e estratégias descritos na introdução e nos casos de uso, embasam uma concepção de educação inclusiva, e possibilidade de gestão educacional com inovação (Moran, 2003, p. 151-161). Além disto, tal convergência contempla a busca da transformação social através da educação em valores, com ensino via formas dialógicas e democráticas de resolução de conflitos cotidianos e dos problemas sociais, com proposta de articulação dos conhecimentos populares com os conteúdos científicos e culturais, pois sem estes saberes, também não haverá cidadania (Araújo, 2003).

Os casos de uso são validados por recomendação de especialistas com base empírica para ensinar a programar desde o início da formação dos indivíduos no ensino básico (Mateas, 2005), aplicar conceitos e padrões relativos ao uso da tecnologia na educação, tal como a combinação entre as tecnologias padrão da Internet e o conceito de objetos educacionais, devido ao seu potencial de reusabilidade, modularidade, generalidade, adaptabilidade e escalabilidade (Ferreira, 2004; Niederauer, 2007, p.17-40). Essas características possibilitam ampliar as habilidades cognitivas e de percepção dos indivíduos, usando como base o conceito de modularidade, que pode ser considerado como um tipo de unificador de conceito científico, pois aprece em vários campos da ciência; biologia, física, psicologia etc. (Jablan, 2005, p. 259).

Assim, pode-se pensar em uma analogia com a sentença anterior e fundamentar a unificação de recursos propiciados pelas TIC em um AV de 2D ou 3D. As TIC propiciam apropriar-se, usar e difundir diversos recursos e técnicas que podem ajudar na expressividade dos indivíduos. Por exemplo, no caso de uso 1 foram usados computador com placa de captura de vídeo, técnicas de edição de imagem em bitmap e MPEG, pesquisa na Internet, cinematografia, arquivos de áudio, colagens, recortes fílmicos, vídeo cassete, aparelho de DVD e software de baixo custo. A montagem do AV teve suporte da VRML que propicia unificar a modularidade dos diversos objetos de aprendizagem em ambiente único, similar aos trabalhos apresentados nas figuras- 1 e 2 .

Embora, o conhecimento em comum sobre técnicas de desenvolvimento de um AV possa ser considerado como uma forma de valor agregado e de influência benéfica na comunicação de M1 e M2, é importante citar que M1 relata não ter tido contribuição no sentido de manipulação direta de arquivos por parte de M2 na construção do AV. Pois, segundo M1, os tempos e espaços de encontro dos dois são reduzidos devido aos horários de trabalho e estudo diversificado de ambos. Assim, avalia-se que as interações face a face no laboratório de informática da escola, e a perseverança de M1 em investigar e desenvolver conhecimento fora do ambiente escolar têm propiciado para M1 aprender a aprender com autonomia em processo de educação continuada e compartilhada, em contexto cognitivo adequado (Minsky, 2006, p. 240-245).

Este resultado valida a tese de que por meio da interação de um estudante com um educador tecnicamente preparado, e com suporte do computador, e arquivos e instrumentos multimídia nas atividades de criar e de programar, a construção de conhecimento pode ser estimulada pela ocorrência do ciclo descrição-execuçãoreflexão- depuração conforme Valente (1993) citado por (Almeida et al, 2003, p. 50, 51).

A confirmação de tal processo se sustenta no relato de M1, que avalia ter melhorado suas habilidades de leitura, escrita e pesquisa, após iniciar seu interesse por tecnologias da Internet. M1 reflete criticamente dizendo que antes quando fazia uma pesquisa na Internet anotava a primeira informação que aparecia, e depois com as orientações recebidas foi percebendo que é necessário entender o contexto. M1 relata que alguns conceitos matemáticos como os de positivo e negativo relativos ao plano cartesiano 
ensinados durante as aulas contribuem para o entendimento de como pensar para construir um AV. Outra estratégia citada é que o desenho feito pelo autor1, no papel, para visualização de informação na primeira interação para aprender VRML, foi fundamental para o entendimento de transformação das figuras geométricas na tela e sua relação com os símbolos X, Y, Z. M1 solicita informações para compreender como produzir e desenvolver uma animação, e na sequiência é realizada uma oficina.

Em 22/06/2007, M1 esteve no laboratório de informática e demonstrou que certas informações recebidas no decorrer do processo de aprendizagem sobre software livre foram importantes para ele. M1 interage com o autor1 por volta de 40 minutos explicando como segue tutoriais do Blender ${ }^{\mathrm{TM}}$ (2007) para modelar personagens. Este software é aplicado nos estudos de modelagem e conservação do patrimônio histórico da cidade de São Paulo (DPH, 2007).

M1 comenta sobre algumas dificuldades de entender a interface do software, devido o instrumento ser uma novidade, e, também, por suas limitações para leitura de textos em língua inglesa. Entretanto, ao acessar o tutorial na Internet e lê-lo de forma colaborativa com o autor1, M1 diz ter compreendido alguns pontos da interface e de uso do sistema que estavam difíceis de entender, pois já havia explorado o software e aprendido alguns conceitos de uso da interface por tentativa e erro. Embora admita que haja dificuldades, M1 engaja-se em modelar rostos e discute durante a leitura do tutorial como mover os vértices de modo a apresentar profundidade ao modelo. Seu conhecimento prático e argumentações de modelagem usando o Blender ${ }^{\mathrm{TM}}$ revelam maior domínio técnico do software do que o do autor1. As habilidades de pesquisa de M1 e busca de novos instrumentos de trabalho são reafirmados quando M1 comenta usar um software chamado Photofiltre ${ }^{\mathrm{TM}}$ (2007), argumentando conscientemente, que ele é mais poderoso que o Paint ${ }^{\mathrm{TM}}$ e menos complicado para produzir seus desenhos do que o Gimp ${ }^{\mathrm{TM}}$.

Outros fatores relevantes, que parecem validar o trabalho apresentado, são sua expansão e aceitação em outra comunidade escolar, e que estudantes de $5^{\circ}$ e $6^{\circ}$ anos têm comparecido ao laboratório da escola Ernani para solicitar cursos de informática relativos à computação gráfica e passam a ter contato com VRML e HTML. Além disso, a participação constante da comunidade escolar nos projetos com mídias interativas de diversas modalidades, viabilizou a participação da escola nas experiências de construção do projeto um computador por criança conforme (Araújo, 2007, 31p.).

\section{Conclusão}

Este artigo apresentou experiências cotidianas de uso de mídias interativas no processo de construção de conhecimento dos indivíduos e de produção de conteúdo digital. As experiências demonstraram ser efetivas, dinâmicas e sustentáveis na formação técnica, pedagógica e humana de educadores e estudantes de modo formal e informal. Elas podem ser replicadas com baixo custo, mas com alta qualidade e dinamismo para o desenvolvimento pessoal e profissional dos indivíduos.

Este processo dinâmico e qualitativo de construção permanente de conhecimento passa pela alfabetização digital dos cidadãos, no sentido de compreender a importância da leitura e escrita tradicionais no desenvolvimento de sua alfabetização digital. Porém, também, indica que os indivíduos nunca estejam restritos a elas, nestes tempos de mudança e de necessidade de muita autonomia e adaptabilidade de todos os Cidadãos.

\section{Agradecimentos}

Agradecemos todos que têm ajudado no desenvolvimento das experiências e elevado a qualidade das interações de ensino-aprendizagem da comunidade escolar Ernani. Que Deus os abençoe sempre.

\section{Referências}

AFONSO, R. S. e SOARES, L. F. G., Desenvolvimento humano e apropriação das TICs In: SANTOS, R. S., (org) CGIbr. Pesquisa sobre o uso das tecnologias da informação e da comunicação no Brasil - TIC domicílios - TIC empresas, 2005,

http://www.cetic.br/tic/2005/indicadores-2005.pdf Acesso em: 08 jul. 2007

ALMEIDA, M. E. B.; MORAES, M. C.; LILAVATI, A. O.; PELLEGRINO, C.; GUIOTI, E.; ARNT, R. M. e ALLEGRETTI, S.

M., Formação de educadores via telemática: relato de uma experiência In: VALENTE, J., (org), A Formação de Educadores para 
o Uso da Informática na Escola, Brasil, Nied, Capitulo 3, 2003, 50-51 p. AMES, A. L.; NADEU, D. R.; MORELAND, J. L., VRML 2.0 sourcebook second edition, Wiley and Sons, USA, 1997. ANDERS, P., (1998) Envisioning cyberspace, designing 3D electronic spaces, USA, McGrawHill, pp. 153-217. ARAÚJO, P., Inclusão digital: cada criança (e professor) com seu laptop. In: GROSSI, G. P. (org) Revista nova escola, São Paulo, ed. Abril, ano XXII, N ${ }^{\text {a }} 23$, Junho/Julho, 2007, 31 p.

ARAÚJO, U. F. Temas transversais e a estratégia de projetos, São Paulo, Moderna, 2003, 58-59 p.

AUGUSTO, T. G. S. e CALDEIRA, A. M. A., Dificuldades para a implantação de práticas interdisciplinares em escolas estaduais, apontadas por professores da área de ciências da natureza. In: MOREIRA, M. A. (Editor) Investigações em ensino de ciências IENCI, Vol. 12, N. 1, março de 2007, Instituto de Física, Universidade Federal do Rio Grande do Sul, Porto Alegre, Brasil. http://www.if.ufrgs.br/ienci/ Acesso em: 08 jul. 2007

BALBONI, M., Apresentação. In: VIEIRA, A. C. G. (org), CGIbr. Pesquisa sobre o uso das tecnologias da informação e da comunicacão no Brasil - TIC domicílios - TIC empresas, 2006, http://www.cetic.br/tic/2006/indicadores-2006.pdf BARRETO, R. G., Novas tecnologias na educação presencial e a distância II, in: BARBOSA, R. L. L. (Org.) Formação de educadores: desafios e perspectivas, Brasil, Ed. Unesp, 2003, 109-117p.

BARROS, P. G. Realidade virtual e multimídia, 2004, http://www.di.ufpe.br/ if124/vrml/vrml.htm Acesso em: 22 jun. 2007 BLENDER TUTORIAL http://www.blender.org/tutorials-help/tutorials/ Acesso em: 10 jul. 2007

BRUTZMAN, D. e DALY, L., X3D - Extensible 3D graphics for web authors, USA, Morgan Kaufmann, 2007, 1-36 p. CABRAL, M.; ZUFFO, M.; GHIROTTI, S.; BELLOC, O.; NOMURA, L.; NAGAMURA, M.; ANDRADE, F.; FARIA, R. e FERRAZ, L., An experience using X3D for Virtual Cultural Heritage, ACM Web3D symposium, Perugia, Italy - April 2007. CASTELLS, M., A Sociedade em rede: a era da informação: economia, sociedade e cultura, Brasil, Paz e Terra, volume 1, 2005, 69 p.

CAVALLO, D., Emergent designing and learning environments: Building on indigenous knowledge, IBM Systems Journal, Vol. 39, NOS 3\&4, 2000. http://www.research.ibm.com/journal/sj/393/part2/cavallo.html Acesso em: 22 jun. 2007BOEHM, B. e EGYED, A., Improving the life-cycle process in software engineering education, Published in the Proceedings of the European Software Day (as part of the 24th Euromicro conference), 1998, http://sunset.usc.edu/ aegyed/publications/Improving_the_LifeCycle_Process_in_Software_Engineering_Education.pdf Acesso em: 22 jun. 2007

CORTONA 3D PAGE OF THE WEEK, 2007, http://www.parallelgraphics.com/products/cortona/best Acesso em: 22 jun. 2007 CUNNINGHAM, S., Programming in OpenGL for visual communication, Pearson Prentice Hall, USA, 2007, $331-395$ p. DEPARTAMENTO DO PATRIMÔNIO HISTÓRICO, http://www.fotoplus.com/dph/modelagem/ Acesso em: 20 jun. 2007 Dolabela, F., Pedagogia Empreendedora, Brasil, Editora de Cultura, 2003, 47-51, 91-93.

DUTRA, R. L. S. e TAROUCO, L. M. R. Objetos de aprendizagem: uma comparação entre SCORM e IMS Learning Design, RENOTE - Revista Novas Tecnologias na Educação, VII Ciclo de Palestras Novas Tecnologias na Educação, 2006 Vol. 4 Nº1 http://www.cinted.ufrgs.br/renote/jul2006/artigosrenote/a1_20138.pdf Acesso em: 22 jun. 2007

FERREIRA, L. F., Usando Objetos Educacionais baseados em Realidade Virtual em ambientes de apoio a construção de conhecimento e aprendizagem de técnicas vídeo cirúrgicas, RENOTE - Revista Novas Tecnologias na Educação, III Ciclo de Palestras sobre Novas Tecnologias na Educação, 2004, http://www.cinted.ufrgs.br/ciclo3/af/14-usandoobjetoseduc.pdf Acesso em: 22 jun. 2007

FRANCO, J. F, e LOPES, R. D., Knowledge Development through Collaborative Work Supported by Interactive Technologies. In Representing and Analyzing Collaborative Interactions: What works? When does is Work? To what extent? 12th International Conference on Artificial Intelligence in Education, AIED Workshop 6, Editor Rosemary Luckin, Amsterdam, 18-22 July, 2005, Holland. http://hcs.science.uva.n1/AIED2005/W6proc.pdf Acesso em: 22 jun. 2007

FRANCO, J. F. Developing Skills teaching and learning using Web standards and interactive 3D Virtual Environments and Multimedia tools - 20th World Conference on Open Learning and Distance Education - Düsseldorf, Germany, 2001. http://www.educorp.futuro.usp.br/publicacoes/Developing\%20Skills.doc Acesso em: 22 jun. 2007

FRANCO, J. F. e LOPES, R. D. Novas Tecnologias em Ambientes de Aprendizagem; Estimulando o Aprender a Aprender, Transformando o Currículo e Ações, RENOTE - Revista Novas Tecnologias na Educação, III Ciclo de Palestras sobre Novas Tecnologias na Educação, 2004. http://www.cinted.ufrgs.br/ciclo3/af/39-novastecnologias.pdf Acesso em: 22 jun. 2007 FRANCO, J. F.; CRUZ, S. R.R. e LOPES, R. D. (2006d) Computer Graphics, Interactive Technologies and Collaborative Learning Synergy Supporting Individuals' Skills Development, The 33 International Conference And Exhibition on Computer Graphics and Interactive Techniques, SIGGRAPH 2006, 30 July - 03 August 2006, Educators Program, Boston Convention and Exhibition Center - Boston Massachusetts - USA http://delivery.acm.org/10.1145/1180000/1179338/p42franco.pdf?key1=1179338\&key2=0205817711\&coll=\&dl=ACM\&CFID=15151515\&CFTOKEN=6184618G Acesso em: 20 jun. 2007

FRANCO, J. F.; FRANCO, N.F.; LOPES, R. D. e CRUZ, S. R. R. (2006a) Apresentando uma arquitetura pedagógica e técnica usada em sinergia com recursos multimídia na construção cooperativa de saberes, RENOTE - Revista Novas Tecnologias na Educação, VII Ciclo de Palestras Novas Tecnologias na Educação, Vol. 4 No 1 - Julho 2006 http://www.cinted.ufrgs.br/renote/jul2006/artigosrenote/a8_21134.pdf Acesso em : 20 jun. 2007 FRANCO, J. F.; MARIZ, L. R.; LOPES, R. D.; CRUZ, S. R. R.; FRANCO, N., F. e DELACROIX, E., (2006b) Desenvolvendo saberes com suporte de tecnologias interativas, arte e cultura em ambientes de ensino-aprendizagem diversos, TecEduc@tion 2006 - $\mathbf{3}^{\circ}$ Congresso Internacional de e-Learning e Tecnologia Educacional, 04 a 06 outubro de 2006, http://www.techne.com.br/humus/ Acesso em: 20 jun. 2007

FRANCO, J.F.; FRANCO, N.F.; CRUZ, S.R.R. e LOPES, R. D., (2006c) Aplicando a Sinergia entre artes, cultura, realidade virtual e computação gráfica interativa na construção colaborativa e interdisciplinar de conhecimento, Publicação: [SVR] VIII: 2006 Maio. 2-5: Belém (PA)- http://www.sbc.org.br/bibliotecadigital/?module=Public\&action=SearchResult\&author=1024 Acesso em: 20 jun. 2007

FRANCO, N.F.; STORI, N., LOPES, R. D. e FRANCO, J. F., Um caso de Construção Colaborativa de Conhecimento e

Desenvolvimento Educacional Interdisciplinar com Mediação das Tecnologias da Informação e da Comunicação Novembro/2005, Revista RENOTE, Vol.3, N², VI Ciclo de Palestras Novas Tecnologias na Educação

http://www.cinted.ufrgs.br/renote/nov2005/artigosrenote/a49_cintednovembro.pdf Acesso em: 22 jun. 2007

FREIRE, P., Pedagogia da autonomia, Brasil, Paz\&Terra, 2004.

GOLDBERG, Projects, 2003, http://www.athomasgoldberg.net/id16.html Acesso em: 22 jun. 2007

HERNÁNDEZ, F., Cultura visual, mudança educativa e projeto de trabalho, Porto Alegre, ARTMED, 2000, 50-51 p.

HIPPEL, E. V., Democratizing innovation, USA, The MIT Press, 2006.

HIRSCHHEIM, R., The Internet-based education bandwagon: look before you leap: Communications of the ACM, July 2005/vol. 48. $\mathrm{n}^{\circ}$ 7, USA, ACM press, 97 -101).

ICEL - INTERNATIONAL CONSORTIUM FOR EXPERIENTIAL LEARNING, 2007 http://www.icel.org.uk/\#el Acesso em: 22 jun. 2007

INAF - INDICADOR NACIONAL DE ANALFABETISMO FUNCIONAL (5o. INAF Relat363rio_03_doc) 
http://www.acaoeducativa.org.br/downloads/inaf05.pdf Acesso em: 22 jun. 2007

JABLAN, S., V., Modularity in Art. In: CALLEBAUT, W., e RASSKIN, G. D., (ed.) Modularity: understanding the development and evolution of natural complex systems, USA, MIT PRESS, 2005, 259 p.

KINER, C., Sistemas de Realidade Virtual, http://www.dc.ufscar.br/ grv/tutrv/tutrv.htm Acesso em: 22 jun. 2007

LABORATÓRIO DE SISTEMAS INTEGRÁVEIS, http://www.lsi.usp.br/interativos/nrv/cv2/home/aplicativo.html Acesso em: 22 jun. 2007

LAYBOURnE, K. e Bell, A., Web animation, in: LAYBOURnE, K., The animation book: new digital edition, New York, Three Rivers Press, 1998, 265-276 p.

LAYBOURNE, K. e GOLDEBERG, A., Improv animation, in: LAYBOURNE, K., The animation book: new digital edition,

New York, Three Rivers Press, 1998, 258-259 p.

LAYBOURNE, K. e JANE, W., Performance animation, in: LAYBOURNE, K., The animation book: new digital edition, New York, Three Rivers Press, 1998, 264 p.

MATEAS, M., Procedural Literacy: Educating the New Media Practitioner. On The Horizon. Special Issue. Future of Games, Simulations and Interactive Media in Learning Contexts, v13, n1 2005

http://www.lcc.gatech.edu/ mateas/publications/MateasOTH2005.pdf Acesso em: 10 jul. 2007

MINSKY, M., Resourcefulness, This is a draft (July 29, 2005) of Part VIII of The Emotion Machine,

http://web.media.mit.edu/ minsky/E8/eb8.html Acesso em: 10 jul. 2007

MINSKY, M., The emotion machine: commonsense thinking, artificial intelligence, and the future of the human mind, USA, Simon \& Schuster, 2006, 166-169, 240-245 p.

MORAN, J. M. C., Gestão inovadora com tecnologias. In: VIEIRA, A. T.; ALMEIDA, M. E. B. e ALONSO, M., Gestão

educacional e tecnologia, Brasil, AVERCAMP Ed., 2003, 151-161 p.

NIEDERAUER, J., Web interativa com ajax e php, Brasil, Novatec, 2007, 17-40 p.

PERLIN, K., 2007, http://mrl.nyu.edu/ perlin/

PHOTOFILTRE http://www.photofiltre-studio.com/news-en.htm Acesso em: 10 jul. 2007

PINHO, M. S. Realidade Virtual como ferramenta de informática na educação, SBIE de 1996

http://grv.inf.pucrs.br/Pagina/Educa/educa.htm Acesso em: 22 jun. 2007

ROUSSOS, M.; JOHNSON, A. E.; LEIGH, J.; BARNES, C. R.; VASILAKIS, C. A. e MOEHR, T. G., Learning and building together in an Immersive virtual world. In Presence vol. 8, n. 3, June 1999, pp. 247 - 263.

http://www.evl.uic.edu/aej/vrais98/vrais98.2.html Acesso em: 22 jun. 2007

RTI, RESPONSE TO INTERVENTION, 2007, http://www.interdys.org/npdf/rti-rev.pdf Acesso em: 22 jun. 2007

SANTIAGO, J. R. S. JR e SANTIAGO, J. R. S., Capital intelectual: o grande desafio das organizações, Brasil, NOVATEC, 2007, 18-50 p.

SENGE, P., Rethinking control and complexity. In: GIBSON, R., (org) Rethinking the future, England, Nicholas Brealey, 1997, 123- $145 \mathrm{p}$.

TABEROSKY, A., Alfabetização e tecnologia da informação e da comunicação. In: TABEROSKY, A. e GALLART, M. S., (org) Contextos de alfabetização inicial, Brasil, Artmed, 2004, 153-164 p.

TAJRA, S.F., Informática na educação, $5^{\circ}$ edição, São Paulo, Ed. Érica, 2001, 127p.

TAPSCOTT, D. e WILLIAMS, A. D., Wikinomics: how mass collaboration changes everything, USA, Portifólio, 2007.

UFRGS, VIRTUAL CAMPOS, http://www.campusvirtual.ufrgs.br/Pagcampus.htm Acesso em: 22 jun. 2007

VALENTE, J. A., Criando ambientes de aprendizagem via rede telemática: experiências na formação de professores para uso da informática na educação. In: VALENTE, J. A., (org.) Formação de educadores para uso da informática na escola, Campinas, 2003, 1-19 p.

VIEIRA, A. T., Sistemas de informação e comunicação: apoio à aprendizagem coletiva na escola. In: VIEIRA, A. T.; ALMEIDA, M. E. B. e ALONSO, M., Gestão educacional e tecnologia, Brasil, AVERCAMP Ed., 2003, 131-150 p.

VRML 2.0 sourcebook on-line examples, http://www.wiley.com/legacy/compbooks/vrml2sbk/cover/cover.htm Acesso em: 22 jun. 2007

WIKIPEDIA, 2007 http://pt.wikipedia.org/wiki/Objeto_de_aprendizagem Acesso em: 22 jun. 2007

YOUTUBE, http://www.youtube.com/results?search_query=charles+chaplin\&search=Search Acesso em: 10 jul. 2007 